\title{
ESTUDIOS
}

\section{¿Es justificable todavía el uso de concepto de verdad en epistemología?}

\author{
Luis Piscoya Hermoza \\ Universidad Nacional Mayor de San Marcos
}

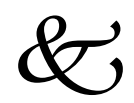

\section{Resumen}

Este artículo prueba suficientemente que ni los deflacionismos ni los consensualismos afectan, hasta la fecha, la sustentabilidad de la convención T de Tarski como herramienta metodológica para la ciencia y la filosofía rigurosa. También explica por qué, propuestas como la de Popper son insatisfactorias debido a que soslayan el carácter monádico del predicado verdadero.

Palabras claves: Satisfabilidad, Metalenguaje, Lenguaje objeto, Enunciado verdadero, Convención T.

\begin{abstract}
Is the use of the concept of truth in epistemology justifiable? In this article the author engages in 1. proving that neither "deflationisms» nor "consensualisms» have affected the validity of Tarski 's T convention as a methodological tool when one gets involved in a rigurous scientific or philosophical work; and 2. explaining why proposals like that of Popper's are unsatisfactory because their overlooking the monadic character of the true predicate.
\end{abstract}

Key words: Metalanguage, Object-Language, True statement; Tarski's T convention, Enunciation, Satisfability. 


\section{Planteamiento del problema}

La presente ponencia asume como un hecho constatable que, durante el siglo pasado, el uso del concepto de verdad para evaluar el conocimiento científico, ha mostrado una tendencia decreciente. Por razones de espacio me excuso de aportar argumentos justificatorios de la afirmación anterior debido a que este punto de vista lo he defendido en un artículo que figura en las actas del VII Congreso Nacional de Filosofía realizado en la Universidad Católica de Lima en 1998. En lo que sigue me propongo inicialmente proporcionar buenas razones para probar que las tesis que han sostenido la eliminabilidad de concepto de verdad en la evaluación de la ciencia, a través del argumento conocido como de la redundancia o de la afirmación desencomillada de un enunciado, son infundadas pese al brillo intelectual de algunos de sus teóricos, entre ellos, F. P. Ramsey, T. Kotarbinski y W. O. Quine. En segundo lugar mostraremos que la concepción semántica de la verdad de Alfredo Tarski constituye el planteamiento teórico más sólido del siglo XX para dar cuenta del uso adecuado de dicho concepto en las ciencias formales, en las factuales o empíricas, en la Epistemología y en el lenguaje cotidiano u ordinario. Para ello probaremos que la modificación a la definición del concepto de verdad de Tarski, propuesta por Popper, es insatisfactoria porque le quita generalidad y reabre una larga discusión, de complejidad comparable a la suscitada por el concepto de verdad, sobre la definición del concepto de hecho. En el mismo sentido probaremos que las teorías consensualistas de la verdad, de aliento pragmatista, de J. Habermas y O. Appel no son alternativas sostenibles a la tesis de Tarski debido a que carecen de consistencia interna o conducen a una problemática difusa, imprecisa y metafísica cuando pretenden definir el concepto de consenso fundado. Finalmente abordamos un tema que Tarski dejó pendiente debido a que, muy probablemente, no consideró pertinente indagar por la justificación de nuestra tendencia a aceptar que existen enunciados que son verdaderos en tanto que son satisfechos por los objetos del mundo externo. Consideramos que esta última problemática, cuya solución presumiblemente ha excedido los intereses de Tarski, ha recibido un tratamiento insatisfactorio desde el punto de 
vista tanto del denominado realismo interno como de los consensualismos.

\section{La pertinencia de la convención T}

Es del caso anotar que cuando se formuló inicialmente la tesis que sostenía la eliminabilidad del predicado verdadero por parte de Ramsey y Kotarbinski todavía no se había demostrado el segundo teorema del Gödel, ni Tarski había formulado su definición del concepto de verdad para los lenguajes formalizados. De conocer Ramsey estos resultados posiblemente hubiera modificado su punto de vista en la medida que ellos probaron la inviabilidad del proyecto formalista. En efecto, de los resultados, complementarios entre sí, de Gödel (1930) y de Tarski (1931) se deduce claramente que el concepto de verdad en la lógica, en la matemática y en las ciencias matematizadas no es reemplazable por el concepto de prueba ni es fácilmente omitible en tanto que a través de la definición de Tarski, denominada por él mismo convención $\mathrm{T}$, se prueba expeditivamente la consistencia de las teorías científicas formalizadas a través de versiones reformuladas del teorema de Löwenheim-Skolem. En el caso de Quine, que conoció estos resultados, su argumento que afirma la trivialidad por redundante de la convención $\mathrm{T}$ parece estar inducido por la simplicidad del conocido ejemplo:

a) El enunciado 'La nieve es blanca' es verdadero si y sólo si, la nieve es blanca.

En este caso es claro que la afirmación 'La nieve es blanca' es verdadera' equivale simplemente a reconocer que la nieve es blanca, lo que es aceptable bajo la presuposición de que todos conocemos el color de la nieve. Sin embargo si tomamos como ejemplo:

b) La primera línea de la Metafísica de Aristóteles es verdadera si, y sólo si, todos los hombres tienden por naturaleza al saber, la aceptación del enunciado nombrado en el definiendum, sin necesidad de afirmar expresamente su verdad, no es tan sencilla como en el caso anterior debido a que el conocimiento del texto de la Metafísica de Aristóteles no es parte de la experiencia cotidiana y a que aún en el caso de que seamos buenos lectores del estagirita, el enunciado Todos los hombres tienden por naturaleza al saber es de aceptabilidad muy 
discutible en relación con la del que describe el color de la nieve. Sin embargo, alguien podría argumentar que si supusiéramos la existencia de una comunidad de peripatéticos altamente informados de la obra de Aristóteles, entonces la omisión del concepto de verdad en el ejemplo $b$ ) sería posible. El argumento anterior da lugar a que para fundamentar nuestra tesis recurramos a un ejemplo que pruebe de manera concluyente que el predicado verdadero no es omitible bajo la suposición de un contexto adecuado. Nos valdremos de uno propuesto por Tarski (1944: 92).

c) Todas las consecuencias de los enunciados verdaderos son verdaderas.

En este caso el cuantificador universal nos permite referirnos a una clase infinita de enunciados verdaderos que no es posible reducir a una conjunción completa de enunciados presentados desencomilladamente.

Es un hecho conocido que la tesis que afirma que el concepto de verdad es omitible por ser redundante no es razonablemente sostenible en las teorías científicas formalizadas en virtud de la validez de los resultados de Gödel y Tarski. No lo es tampoco en todos los casos, como el ejemplo c), en los que mediante el lenguaje ordinario, empleado como metalenguaje intuitivo, formulamos metaenunciados cuantificados universalmente para hablar de clases no finitas de enunciados verdaderos. En estos casos, de asumir la tesis de la redundancia omitible, que es una forma de deflacionismo, renunciaríamos al uso de los criterios específicos de satisfabilidad, propios de las comunidades científicas, que nos permiten descartar a los enunciados que no pueden formar parte de una teoría contrastable. $\mathrm{Y}$ es ciertamente más compatible con nuestro punto de vista pensar que es la satisfabilidad la que sustenta la aceptabilidad que la inversa.

\section{Verdad como satisfabilidad}

En este apartado trataremos de justificar la tesis que sostiene que la definición del concepto de verdad para los lenguajes formalizados aportada por Tarski constituye el planteamiento más riguroso del siglo XX para explicar satisfactoriamente el uso del concepto de verdad en 
la ciencia y en las argumentaciones que tienen algún tipo de pretensión de validez, como es el caso de las filosóficas. El mismo Tarski ha señalado que su definición está construida dentro de lo que él califica como concepción semántica, entendiendo a la semántica como una disciplina que estudia las relaciones entre las expresiones de un lenguaje y los objetos o estados de cosas a los que se refieren estas expresiones (Tarski, 1944: 72).

Los conceptos centrales de la semántica tarskiana son los de designación, definición, satisfacción y verdad. Sin embargo, solamente los tres primeros pueden ser entendidos como predicados relacionales de dos posiciones que establecen un vínculo entre expresiones y objetos. Para probar que las definiciones cumplen también esta función recurre al caso en el que una ecuación con raíz única del tipo $2 \cdot x=1$ define unívocamente al objeto $1 \frac{1}{2}$. El concepto de verdad, a diferencia de la concepción tradicional basada en la clásica expresión latina adaequatio intellectus et rei, en este contexto, no establece una relación entre lo dicho o pensado y las cosas porque, simplemente, es un predicado de una posición que se aplica solamente a un enunciado que es definido en términos de la relación de satisfacción que es, propiamente, el predicado relacional que establece un vínculo entre las expresiones y los objetos a los que ellas se refieren.

En consecuencia, el concepto de verdad, desde esta perspectiva, es semántico no por establecer un vínculo entre expresiones y objetos sino por estar definido en términos del concepto semántico de satisfacción. El argumento anterior marca una diferencia teórica entre las tesis de Tarski y las tradicionales tesis de la correspondencia aunque ello no ha sido enfatizado suficientemente por dicho autor. Sin embargo, en esta distinción puede encontrarse una primera explicación a la renuencia de Tarski a introducir en su definición el concepto de correspondencia como lo han sugerido, entre otros, Popper.

Es sabido que la definición del concepto de verdad, en su versión rigurosa, contenida en su trabajo publicado en polaco, en una versión restringida presentada por J. Lukasiewicz en 1931 (Tarski, 1956: 152), fue construida dentro del lenguaje formalizado del cálculo de las clases, como un ejemplo que posibilita la aplicación de esta metodología a otras áreas. Como puede observarse en la cita siguiente, tomada de la 
versión inglesa titulada Concept of truth in formalized languages, tal definición carece de la apariencia de simplicidad y obviedad que presentan las divulgaciones de esta definición realizadas por el mismo Tarski.

Definition 23. $x$ is a true sentence -in symbols $x \in \operatorname{Tr}$ - if and only if $x \in$ $S$ and every infinite sequence of classes satisfies $x$. (Tarski, 1956: 195)

En tanto que la definición anterior fue formulada en un contexto de investigación en el que los resultados del primer informe sólo eran válidos sin restricciones para lenguajes de orden finito, conviene en este caso recurrir al resumen que hizo el propio Tarski de las modificaciones que hizo, para superar las dificultades antes anotadas, en un Postscript (1936). En dicho resumen ya no aparece limitación alguna respecto del orden finito o no del lenguaje y sólo se recurre al orden del metalenguaje que debe ser igual o mayor que el del lenguaje objeto. De esta manera se enfatiza aquello que se ha divulgado en términos de que la riqueza expresiva del metalenguaje debe ser mayor que la del lenguaje objeto de tal suerte que la semántica del lenguaje objeto pueda estar incluida en las sintaxis del metalenguaje que, en la cita que a continuación hacemos, es denominada morfología.

a) For every formalized language a formally correct and materially adequate definition of true sentence can be constructed in the metalanguage with the help only of general logical expressions, of expressions of the language itself, and of terms from the morphology of language -but under the condition that the metalanguage possesses a higher order than the language which is the object of investigation.

b) If the order of the metalenguage is at most equal to that of the language itself, such a definition cannot be constructed. (Tarski, 1956: 273)

\section{El carácter contextual de la convención T}

La versión simplificada de la denominada por su autor convención $T$ es la siguiente equivalencia:

(T) $X$ es verdadera si, $y$ sólo si, $p$.

Utilizaremos la equivalencia $T$ en tanto que facilita la explicación de las características fundamentales de la definición del concepto de 
verdad de Tarski. El esquema $T$ no pretende ser una definición del concepto de verdad sino del concepto de oración, proposición o enunciado verdadero en un lenguaje objeto dado $\mathrm{L}$, lo que debe entenderse, además, como una definición parcial del concepto de verdad para dicho lenguaje L. La definición completa del concepto de verdad para el lenguaje finito L estaría dada por la conjunción lógica que tiene como cada uno de sus componentes a cada uno de los reemplazos realizados con los nombres de las proposiciones de $\mathrm{L}$ en lugar de $X$ y con el texto de cada una de dichas proposiciones de L en reemplazo sucesivo de $p$.

Tarski prueba que la definición anterior es formalmente correcta y materialmente adecuada. Lo primero debido a que sólo es aplicable a lenguajes-objeto formalizados describibles mediante metalenguajes con cuyos medios expresivos es posible hablar de la verdad de las oraciones de los lenguajes-objeto sin incurrir en contradicción lógica. Lo segundo debido a que la convención $\mathrm{T}$ posibilita realizar para un lenguaje $\mathrm{L}$ tanto definiciones parciales como totales del concepto de verdad siempre que el metalenguaje de $\mathrm{L}$ sea de un orden mayor que el de $\mathrm{L}$.

El ejemplo más conocido que revela incorrección formal de la paradoja del mentiroso o de Epiménides, contradicción construible en los lenguajes naturales debido a que éstos no distinguen entre lenguajeobjeto y metalenguaje. Esta peculiaridad da lugar a que Tarski los califique de lenguajes semánticamente cerrados y los descalifique para el uso científico debido a que la inconsistencia que es construible en ellos, desde su punto de vista, es una propiedad que invalida a un lenguaje como medio de expresión científica.

Con respecto al concepto de satisfacción, su uso en la convención T, en su versión simplificada, está resuelto por la presencia de $p$. Sin embargo, ello resulta insuficiente si consideramos que la solución dada por Tarski al uso científico del concepto de verdad gravita decisivamente sobre el significado del concepto de satisfacción en el definiens de la convención $\mathrm{T}$, lo que transfiere la solución final a los criterios imperantes en cada comunidad científica. Adicionalmente, es importante anotar que en numerosos trabajos en lógica se invierte el sentido del uso tarskiano. Esto es, el concepto de satisfacción se define en términos 
del concepto de verdad, procedimiento que presupone al concepto de verdad como concepto no definido. Sin embargo, por razones que no es el caso detallar en esta ponencia, pensamos que la decisión metodológica tarskiana de introducir el concepto de verdad como concepto definible es filosóficamente más esclarecedora y metodológicamente más productiva. En esta dirección, Tarski asume que dada la función proposicional $\mathrm{P}(\mathrm{x})$, donde $\mathrm{P}$ es el predicado '... es el menor número entero positivo' y dada la función proposicional $\mathrm{Q}(\mathrm{x})$, donde $\mathrm{Q}$ '... es el mayor entero positivo', toda miembro de la comunidad matemática sabe que el número 1 es el único objeto que satisface a la primera y que no existe número alguno que satisfaga la segunda. Consecuentemente, aplicando la definición 23 (antes transcrita) resulta claro que la proposición ' $(\exists x) P(x)$ ' es verdadera y que ' $(\exists x) Q(x)$ ' es falsa.

En general, Tarski apela a la validez del principio del tercio excluido y sostiene que una proposición $p$ es o verdadera o falsa. En el primer caso $p$ es satisfecha por todos los objetos y $\sim p$ no es satisfecha por objeto alguno. Obviamente en el caso en el que $\sim p$ sea satisfecha por todos los objetos $p$ es falsa. Para un análisis más detallado del concepto de satisfacción es necesario recurrir al concepto de interpretación, expediente que omitiremos debido a que para los fines de lo que queremos probar ha sido suficiente aclarar el funcionamiento del concepto de satisfacción en el definiens de la convención $\mathrm{T}$ y mostrar que decidir si estamos o no ante un definiens aceptable es generalmente un procedimiento complejo que corre a cargo de cada comunidad científica.

Por ejemplo, la definición siguiente ' $\leftrightarrow x_{1} \exists x_{2} x_{2} \subseteq x_{1}$ ' si y sólo si, para toda clase b hay una clase a tal que $a \subseteq b$ (Tarski, 1956: 190) es utilizable dentro del cálculo de clases. Sin embargo cualquier persona no está en condiciones de decidir su aceptabilidad o pertinencia como sí ocurre en el famoso caso de la proposición 'la nieve blanca'. Para saber si se cumple o no la condición de satisfacción hay que pertenecer a la comunidad matemática y conocer que el conjunto vacío está incluido en todos los conjuntos. Este conocimiento del contexto es materia del manejo de una semántica especializada, lo que explica que Tarski sostenga la no existencia de un criterio general de verdad. 


\section{Popper y los consensualistas}

Popper en el capítulo 9 de Conocimiento objetivo se propone, por decirlo así, hacer más pedagógica la convención $T$ en base a su experiencia docente, introduciendo la siguiente variante:

$X$ corresponde a los hechos si, y sólo si, p. (Popper, 1992: 288)

De este modo pretende convertir, sin desnaturalizarla, en más inteligible la convención T y en más acorde con la presumible intención de la definición de Aristóteles. El resultado no ha sido el esperado por Popper. En breve, para Tarski el concepto de verdad no expresa una relación entre objetos sino una propiedad especial de un objeto, en este caso, una oración o enunciado. Asimismo, si se introduce el concepto de hecho, la convención T sería aplicable sólo en el nivel factual y se tornaría muy discutible en el nivel de la matemática y de la lógica.

Habermas en un artículo publicado originalmente el 1973 bajo el título Wahrheitstheorien (ver en Nicolas y Frápoli, 1997: 543-595) defiende con detalle lo que el denomina la teoría consensualista de la verdad. Define al concepto de verdad dentro del marco del discurso argumentativo como una pretensión de validez entre cuatro posibles pretensiones. Las restantes son las de inteligibilidad, veracidad y rectitud. Lo que tipificaría a la verdad es que es una pretensión de validez que tiene como justificación el consenso fundado. A su vez el consenso fundado se justificaría a través de los esquemas mentales que ha internalizado la especie humana a través de su evolución, como, según Habermas, lo ha señalado certeramente Piaget.

La dificultad de esta tesis es que si el fundamento último de la verdad es un producto de la evolución de la especie humana en su interacción con el ambiente, entonces el fundamento de los enunciados verdaderos no es estrictamente el consenso sino un mecanismo de la selección natural. Por consiguiente, o la teoría de Habermas no es consensualista en el sentido enfatizado por el título del artículo, o lo es y entonces el proceso evolutivo se convertiría en una instancia explicativa pero no justificatoria. De admitirse esta segunda posibilidad la tesis de Habermas no sería necesariamente irreconciliable con la concepción semántica de Tarski pero tendría la limitación 
epistemológica de establecer fronteras muy difusas entre fundamento, justificación y explicación, lo que reduciría la pretensión de validez de verdad a un consenso fundado para los actores pero no necesariamente para los espectadores. A la anterior insuficiencia tendríamos que añadir que el aporte de Habermas, tal como está expresado en el artículo citado, no contribuye a la solución de problema alguno en los campos de la ciencia, la metodología y la filosofía. En el mejor de los casos contribuiría a entenderlo pero no a resolverlo en términos operativos.

El filósofo Otto Apel, que se ubica también dentro de las orientaciones consensualistas en un artículo titulado iHusserl, Tarski o Peirce? Por una teoría semiótico trascendental de la verdad como consenso (ver Nicolas y Frápoli, 1997: 597-616) defiende la tesis de que el concepto de verdad aplicado a enunciados se justifica, siguiendo a Peirce, como una idea reguladora que se orienta hacia el logro de un consenso ideal y último dentro de una comunidad ilimitada de investigadores. Según Apel esta concepción supera las limitaciones de la teoría fenomenológica de la verdad que la define como evidencia, las limitaciones de la concepción semántica de Tarski y las del coherentismo de Hegel. Lo primero debido a que Husserl no consideró la relevancia del contexto linguístico en la definición del concepto de verdad y se limitó a una noción de evidencia intuitiva desconectada de la acción comunicativa. Lo segundo debido a que Tarski resuelve los aspectos sintácticos y semánticos de la definición del concepto de verdad pero no aborda la dimensión pragmática que requiere una especial consideración de los signos indexicales que sólo tienen significado dentro de un contexto pragmático.

Términos como 'éste', 'aquî', 'otro', etc sólo son inteligibles en el contexto de la acción comunicativa y, por tanto, no deberían ser ignorados, como lo hace Tarski y Carnap, como elementos que forman parte del metalenguaje pragmático que es el que orienta, finalmente, las aplicaciones de cualquier lenguaje-objeto. Lo tercero se debe a que Hegel, que habría sostenido una tesis coherentista, no entendió suficientemente la función semiótica de los términos indexicales que son justamente los que nos permiten establecer una diferencia clara entre el discurso que se refiere al mundo real y el que se refiere a mundos posibles. El pragmatismo de Peirce habría superado las 
deficiencias anteriores al rescatar el valor de los términos indexicales, al enfatizar la conveniencia del uso de condicionales contrafácticos para analizar las experiencias posibles que cabe esperar y al destacar el falibilismo de los consensos reales como un hecho que nos permite entender la no realizabilidad del consenso ideal. La debilidad mayor del punto de vista de Apel es que hace descansar el uso del concepto de verdad en la aspiración de los investigadores a un consenso ideal que es, simplemente, una entidad metafísica a la cual se puede aplicar expeditivamente la Navaja de Occam con cargo a fundamentar el concepto de verdad en alguna forma de vínculo entre el lenguaje y el mundo externo.

\section{La imposibilidad de un criterio general de verdad}

Respecto de nuestra tesis que sostiene que la concepción semántica de la verdad de Tarski es la conceptualización teórica sobre el tema más fuerte del siglo XX, en esta sección nos referiremos a sus insuficiencias epistemológicas y ontológicas que probablemente no son de especial relevancia desde el ángulo del quehacer lógicomatemático pero si desde la perspectiva filosófica. Considero que la conocida tesis de Tarski que afirma que no existe criterio general alguno de verdad puede ser traducida en términos de que no existe criterio general alguno para decidir si cualquier función proposicional es o no satisfacible en un dominio de objetos que forman parte del mundo real o de alguna estructura matemática. Los criterios de satisfabilidad que pueden ser utilizados, por ejemplo, en una interpretación que tiene como dominio un conjunto de bacterias con propiedades bioquímicas son muy diferentes a los que podrían utilizarse en una interpretación que tiene como dominio unidades de un sistema monetario y propiedades económicas. Sin embargo el hecho de que no se pueda arbitrar un criterio general no implica que no se pueda hacer afirmación relevante alguna en este nivel de análisis.

Tener conciencia lúcida de que la aceptación o rechazo de si una función proposicional es o no satisfacible corre siempre a cargo, salvo los casos obvios, de una comunidad especializada es ya un avance. Sin embargo es posible dar un paso más hacia delante para superar las 
limitaciones de los consensualismos radicales y de los pragmatismos restringidos a la predictibilidad y al éxito. En ese sentido puede pensarse en algún principio ontológico que garantice que las expresiones de nuestros lenguajes científicos y naturales hacen referencia a un mundo externo. La acción comunicativa puede ser un buen punto de partida en esta dirección en tanto que presupone que los interlocutores hablan de objetos, procesos o hechos externos a sus mentes lo que incluye la externalidad material de los textos y fonemas a través de los cuales se comunican.

En otro caso, la única hipótesis que explicaría el hecho de la comunicación sería la que sostendría que los interlocutores leen recíprocamente sus mentes, posición metafísica insostenible que no sólo haría innecesario el mundo sino también el lenguaje. El acuerdo, asimismo, presupone para ser aceptado que los interlocutores están hablando de lo mismo y que cada uno de ellos está dotado de voluntad independiente de la del otro. Si esto último no se cumpliera, el consenso no costaría trabajo alguno en tanto que estaría eliminada, en principio, la posibilidad de discrepar. Para descartar una posición extrema como la de Stace (1965) en su defensa de idealismo anotaremos que incurre en un error atribuible también a los consensualistas que presuponen que toda afirmación verdadera es lógicamente argumentable, lo que en el caso de Stace significa claramente, lógicamente demostrable. Sin embargo, la afirmación El mundo existe independientemente de que sea percibido por sujeto cognoscente alguno puede ser verdadera y, en principio no demostrable como lo posibilitan los resultados de Gödel.

Lo expuesto nos permite afirmar que los internalismos de todas las cepas son insostenibles y que la definición de Tarski es utilizable dentro de un contexto en el que estén presentes las teorías lógicas vigentes sobre la construcción de lenguajes formales y una ontología realista que nos asegure que al menos las ciencias fácticas formalizadas siempre pueden ser dotadas de una semántica realista. Ciertamente, el realismo de la definición del concepto de verdad de Tarski, como él mismo lo ha enfatizado, es próximo a Aristóteles pero no necesariamente al criterio de correspondencia que, además, Aristóteles no usó en su famosa definición del Libro Primero de la Metafísica. De lo argumentado, se deduce que menos aún debilitan nuestro punto de vista las objeciones 
derivadas de las teorías que han enfatizado los condicionamientos culturales y neurológicos de la percepción en tanto que nos es suficiente probar que conocemos el mundo en alguna forma y desde alguna parte y que sólo al ojo de Dios le correspondería conocerlo de una sola vez y desde ninguna parte, por usar una metáfora grata a Hilary Putnam.

\section{Referencias bibliográficas}

APEL, KARL, OTTO (1995): ¿Husserl, Tarski o Peirce? Por una teoría semiótico trascendental de la verdad como consenso. En Juan Antonio Nicolás y María José Frápoli (3), pp. 597-616. Versión original inédita (al menos hasta 1995): ¿Huserl Tarski oder Peirce? Für eine trazendentalsemiotische Konsenstheorie der Wahrheit.

HABERMAS, Jürgen (1973): Teorías de la verdad, en J. A. Nicolás y M. J. Frápoli (3), pp. 543-595. Versión original: Wahrheitstheorien (1973) en Fahrenbach, H. (editor) Wirklichkeit und Reflexion, Neske, Pfullingen, pp. 211-265.

NICOLÁS, Juan Antonio y FRÁPOLI, María José, Editores (1997): Teorías de la verdad en el siglo XX. Ed. Tecnos S.A., Madrid.

PISCOYA, Luis (2000): El uso decreciente del concepto de verdad en la epistemología contemporánea, contenido en Miguel Giusti, Editor (2000) La filosofía del siglo XX: balance y perspectivas, Fondo Editorial, Pontificia Universidad Católica del Perú, Lima, pp. 163-174.

POPPER, Karl (1992): Conocimiento objetivo. Ed. Tecnos S.A., Madrid.

PUNTEL, Bruno Lorenz (1993): Wahrheitstheorien in der neuren Philosophie, Wissenschaftliche Buchgesellschaft, Darmstadt.

PUTNAM, Hilary (2001): La trenza de tres cabos. Siglo Veintiuno de España Editores, Madrid.

RORTY, Richard (2000): Verdad y progreso. Ediciones Paidos Iberica S.A., Barcelona.

STACE, Walter (1965): La refutación del realismo, contenido en Salazar Bondy, Augusto (1965) Lecturas filosóficas, Ed. Educación Renovada, Lima, pp. 452-461. La versión original se titula The refutation of realism, Revista Mind (1934). Posteriormente apareció en el libro del mismo autor Modern introduction to philosophy (1963), Free Press of Glencoe Inc. 
LuIS PISCOYA

TARSKI, Alfred (1944): La concepción semántica de la verdad y los fundamentos de la semántica. En Nicolás y Frápoli (3) pp. 63-108. La version original fue The semantic conception of truth and the foundations of semantics, publicada en Philosophy and Phenomenological Research, IV, 1944, pp. 341-375.

(1956): The concept of Truth in formalized languages, publicado junto a otros dieciséis artículos en Logic, Semantics, Methamathematics, papers from 1923 to 1938 by Alfred Tarski (1956), Oxford at The Clarendon Press, traducción de J. H. Woodger, pp. 152-278. Una primera versión de este artículo fue presentada por J. Lukasiewicz a la Sociedad de Ciencias de Varsovia en 1931. La primera publicación en polaco apareció en 1933. La traducción alemana que incluyó un Postscrip se publicó en 1936 bajo el título Der Wahrheitsbegriff in den formalisierten Sprachen contenida en Studia Philosófica, Vol. 1 (1936). La traducción de Woodger se basa en esta última versión.

(1969): Verdad y prueba. Publicado en Luis Piscoya (2000); Tópicos en epistemología, Fondo Editorial, Universidad Inca Garcilaso de la Vega, Lima, pp. 191-230. Traducción de L. Piscoya. La versión original es Truth and Proof, Scientific American, June 1969, Vol. 220, N 6, pp. 63-77. 